\title{
Clutter cancellation in passive radar using batch-based CLEAN technique
}

\author{
Xia Bai* (D), Jiatong Han, Juan Zhao, Yuan Feng and Ran Tao
}

*Correspondence: bai@bit.edu.cn School of Information and

Electronics, Beijing Institute of Technology, 5 South

Zhongguancun Street, Haidian

District, 100081 Beijing, China
Springer Open

\section{Introduction}

Passive radar (PR) is a special bistatic radar system, which exploits signals of opportunity (e.g., FM, DVB-T, GSM, Wi-Fi, etc.) designed for other applications [1-3]. At the last years, lots of literatures on passive radar have been published worldwide. PR systems have received much interest in military as well as commercial applications, mainly because of their covertness, low-cost implementation, and immunity to jamming, etc. [4-7].

A typical PR system consists of two receive channels. One receive channel would sample the signal received directly from the transmitter of opportunity. This is known as the "reference" channel. The other "surveillance" channel is used to detect echoes from targets. The surveillance channel also contains the small fraction of the direct signal and other stationary clutter. In general, the clutter causes serious degradation of dynamic range and masking of weak targets by sidelobes. It is great significance for passive radar signals processing to study clutter cancellation. Now, many solutions have been put forward and can be divided into two categories: (1) adaptive filtering methods and (2) fixed coefficient methods. Some evaluation of clutter cancellation methods also have been presented [8-12]. The adaptive filtering methods mainly included least mean squares (LMS), recursive least squares (RLS), and least square lattice (LSL). These adaptive techniques can

(c) The Author(s). 2021 Open Access This article is licensed under a Creative Commons Attribution 4.0 International License, which permits use, sharing, adaptation, distribution and reproduction in any medium or format, as long as you give appropriate credit to the original author(s) and the source, provide a link to the Creative Commons licence, and indicate if changes were made. The images or other third party material in this article are included in the article's Creative Commons licence, unless indicated otherwise in a credit line to the material. If material is not included in the article's Creative Commons licence and your intended use is not permitted by statutory regulation or exceeds the permitted use, you will need to obtain permission directly from the copyright holder. To view a copy of this licence, visit http://creativecommons.org/licenses/by/4.0/. 
automatically adjust to time-varying signal statistics [10]. Fixed coefficient filtering techniques assume that the clutter terms are fixed throughout the coherent processing interval (CPI). The sample matrix inversion (SMI) [12] is a typical coefficient filtering method. The filter output using the SMI technique in matrix form can be calculated by least squares (LS). In particular, the LS algorithm is very attractive due to its simplicity. Note that the cancellation performance of the fixed coefficient algorithms degraded significantly for time-varying scenario. Clearly, the selection of an appropriate method requires careful consideration of various factors according to the actual circumstance.

CLEAN is another commonly used fixed coefficient filtering method. It was initially developed as a deconvolution tool in the mid-1970s [13, 14]. Recently, some CLEAN-type algorithms have been widely employed to remove the sidelobe effect for the radar signal processing. The CLEAN can be carried out in the range-compressed domain [15-17] or the time domain $[18,19]$. The latter iteratively removes the direct signal and the echoes of stationary objects from the surveillance signal. The CLEAN technique is very similar to matching pursuit (MP), which chooses at each iteration an atom from the dictionary that is best adapted to approximate the residual [20]. The aim of MP is to build a sparse decomposition using an iterative procedure, which has been used in a wide variety of application areas $[21,22]$. In this paper, we introduce an extension of the MP to pursue efficiency of clutter cancellation for passive radar. Our approach named GMP is literally a generalization of the MP in the sense that multiple atoms are identified per iteration and coefficients are estimated by use of LS. Based on GMP and fast Fourier transform (FFT), a batch-based CLEAN technique with a reasonable memory requirement and computational time is proposed. It can identify and remove the largest components of clutter at each iteration; in other words, the clutter cancellation method has a batch processing style.

This paper is organized as follows. In Section 2, the passive radar clutter signal model and CLEAN concept are described. In Section 3, we present GMP and discuss about how to carry out batch-based CLEAN. In Section 4, the performance of the proposed technique is verified by using simulated and real passive radar data. The last section concludes this paper.

\section{Model and basis of CLEAN}

\subsection{Passive radar signal model}

Typical passive radar geometry is depicted in Fig. 1. The transmitter illuminates a target and the reference antenna directs towards it. The surveillance antenna directs towards the target. We assume that the target moves with a given velocity, and $s(t)$ is the transmit signal. Meanwhile, echoes of the reference channel and surveillance channel are denoted as $s_{\text {ref }}(t)$ and $s_{\text {surv }}(t)$, respectively.

Since, at the reference channel, the direct signal is received by the main lobe of the receiver antenna, it is possible to assume that the signal collected by the reference channel does not contain clutter. In other words, the scatterer contributions received from the sidelobes are usually negligible. And then, the reference signal can be written as

$$
s_{\text {ref }}(t)=A_{r} s\left(t-\tau_{r}\right)+n_{\text {ref }}(t)
$$

where $A_{r}$ and $\tau_{r}$, respectively, are the complex amplitude and delay for the direct signal. The amplitude $A_{r}$ represents propagation effect, and the delay $\tau_{r}$, which equals to the 


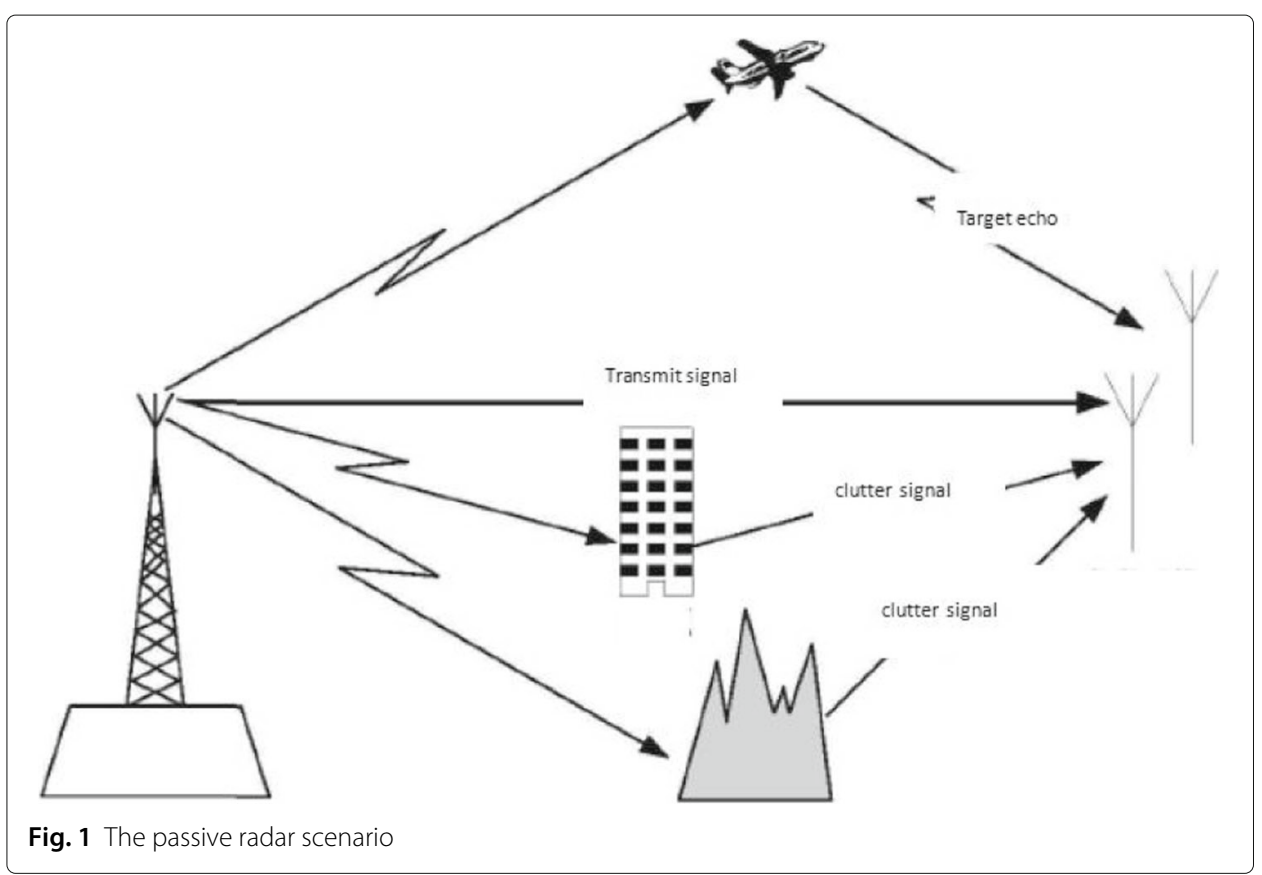

bistatic baseline range over the speed of light, is generally assumed to be known and fixed. $n_{n e f}(t)$ is the thermal noise. A more general model of the reference signal with noise and multipath interference is beyond the scope of this paper, and the detailed description can be found in $[23,24]$.

Assuming that there is only one target, the signal collected by the surveillance channel can be expressed as

$$
s_{\text {surv }}(t)=s_{\text {target }}(t)+s_{\text {clutter }}(t)+n_{\text {surv }}(t)
$$

where

$$
\begin{aligned}
& s_{\text {target }}(t)=A_{m t} s\left(t-\tau_{m t}\right) \exp \left(j 2 \pi f_{m t} t\right) \\
& s_{\text {clutter }}(t)=\sum_{i} A_{i} s\left(t-\tau_{i}\right)=\sum_{i} s_{c i}(t)
\end{aligned}
$$

The complex amplitude, delay, and Doppler shift for the moving target are $A_{m t}, \tau_{m t}$, and $f_{m t}$, respectively. Similarly, the $i$ th path (direct or multipath) $s_{c i}(t)$ results in its own unique complex amplitude $A_{i}$ and delay $\tau_{i} . n_{\text {surv }}(t)$ is the thermal noise at the surveillance antenna.

To obtain target Doppler and range information, cross ambiguity function (CAF) between the surveillance and reference signals is an effective tool. The definition of CAF is

$$
s_{C A F}\left(\tau, f_{d}\right)=\int_{T_{\text {int }}} s_{\text {surv }}(t) s_{\text {ref }}^{*}(t-\tau) \exp \left(-j 2 \pi f_{d} t\right) d t
$$

where $T_{\text {int }}$ is the coherent processing interval (CPI), $\tau$ is the delay, $f_{d}$ is the Doppler frequency, and $*$ denotes the complex conjugate. By substituting Eq. (2) into Eq. (3), Eq. (3) can be approximated as follows:

$$
\begin{aligned}
s_{C A F}\left(\tau, f_{d}\right) & =s_{C A F, \text { target }}+s_{C A F, \text { clutter }}+s_{C A F, \text { noise }} \\
& \approx s_{C A F, \text { target }}+s_{C A F, \text { clutter }}
\end{aligned}
$$


where

$$
\begin{aligned}
& s_{C A F, \text { target }}\left(\tau, f_{d}\right)=\int_{T_{\text {int }}} s_{\text {target }}(t) s_{\text {ref }}^{*}(t-\tau) \exp \left(-j 2 \pi f_{d} t\right) d t \\
& s_{C A F, \text { clutter }}\left(\tau, f_{d}\right)=\sum_{i} \int_{T_{\text {int }}} s_{c i}(t) s_{\text {ref }}^{*}(t-\tau) \exp \left(-j 2 \pi f_{d} t\right) d t
\end{aligned}
$$

It was concluded that the final range-Doppler surface can be written as the sum of the range-Doppler surfaces of each component. In many cases, the clutter signal is much stronger than the useful echo signal. Thus, without compensation, the sidelobe of these

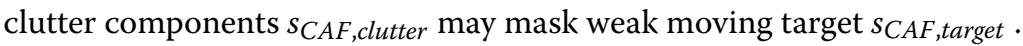

\subsection{CLEAN processing}

MP can be viewed in the radar context as a CLEAN algorithm: estimate iteratively the strongest components of the signal (in the correlation magnitude sense) and remove them from the original signal. We will then provide a brief description.

The goal of clutter cancellation is to remove $s_{\text {clutter }}(t)$ from the surveillance channel signal $s_{\text {surv }}(t)$ by first estimating the unknown clutter $s_{\text {clutter }}(t)$ and then subtracting this estimated component. From Eqs. (1) and (2), we can obtain

$$
s_{\text {clutter }}(t)=\sum_{i} s_{c i}(t)=\sum_{i} w_{i} s_{\text {ref }}\left(t-\tau_{i}^{\prime}\right)
$$

where $w_{i}=A_{i} / A_{r}$ and $\tau_{i}^{\prime}=\tau_{i}-\tau_{r}$ is the relative discrete time delay of the $i$ th path between the reference channel and surveillance channel.

The CLEAN algorithm for the passive radar, as depicted in Fig. 2, is an iterative procedure using the following update formula:

$$
s_{\text {surv }}^{(k)}(t)=s_{\text {surv }}^{(k-1)}(t)-s_{\text {ci_max }}(t)
$$

where superscript $k$ denotes the iteration index, $s_{\text {surv }}^{(0)}(t)=s_{\text {surv }}(t) \cdot s_{\text {ci_max }}(t)=$ $w_{p} s_{r e f}\left(t-\tau_{p}^{\prime}\right)$ is the strongest component of clutter in the surveillance channel at present step. After the peak selection using cross-correlation calculation of the surveillance and reference signals, $\tau_{p}^{\prime}$ and $w_{p}$ can be estimated during each iteration. $s_{s u r v}^{(k)}(t)$ is the "CLEANed" signal of $s_{\text {surv }}^{(k-1)}(t)$ in $k$ th iteration. The process is executed repeatedly until a particular stopping criterion is met.

\section{Batch-based CLEAN method}

In this section, the discrete-time signal model of radar echo will be explained here as an introduction to the problem of clutter cancellation. Then, we extend the concept of MP to a generalization case and use it as a basis for batch-based CLEAN.

\subsection{Vector-matrix form of clutter cancellation}

In case of a digital receiver system, we consider the discrete form of the clutter sampled at interval $T_{s}$, such that $t=n T_{s}, n$ is time index. From Eq. (5), the clutter term $s_{c l u t t e r}(n)$ can be regarded as the weighted sum of all versions of reference $s_{r e f}(n)$ with different time delays. Considering that the moving target term and noise term are usually less significant, the discrete form of surveillance signal can be approximated as

$$
s_{\text {surv }}(n) \approx \sum_{i=0}^{L-1} s_{c i}(n)=\sum_{i=0}^{L-1} w_{i} s_{r e f}(n-i)
$$




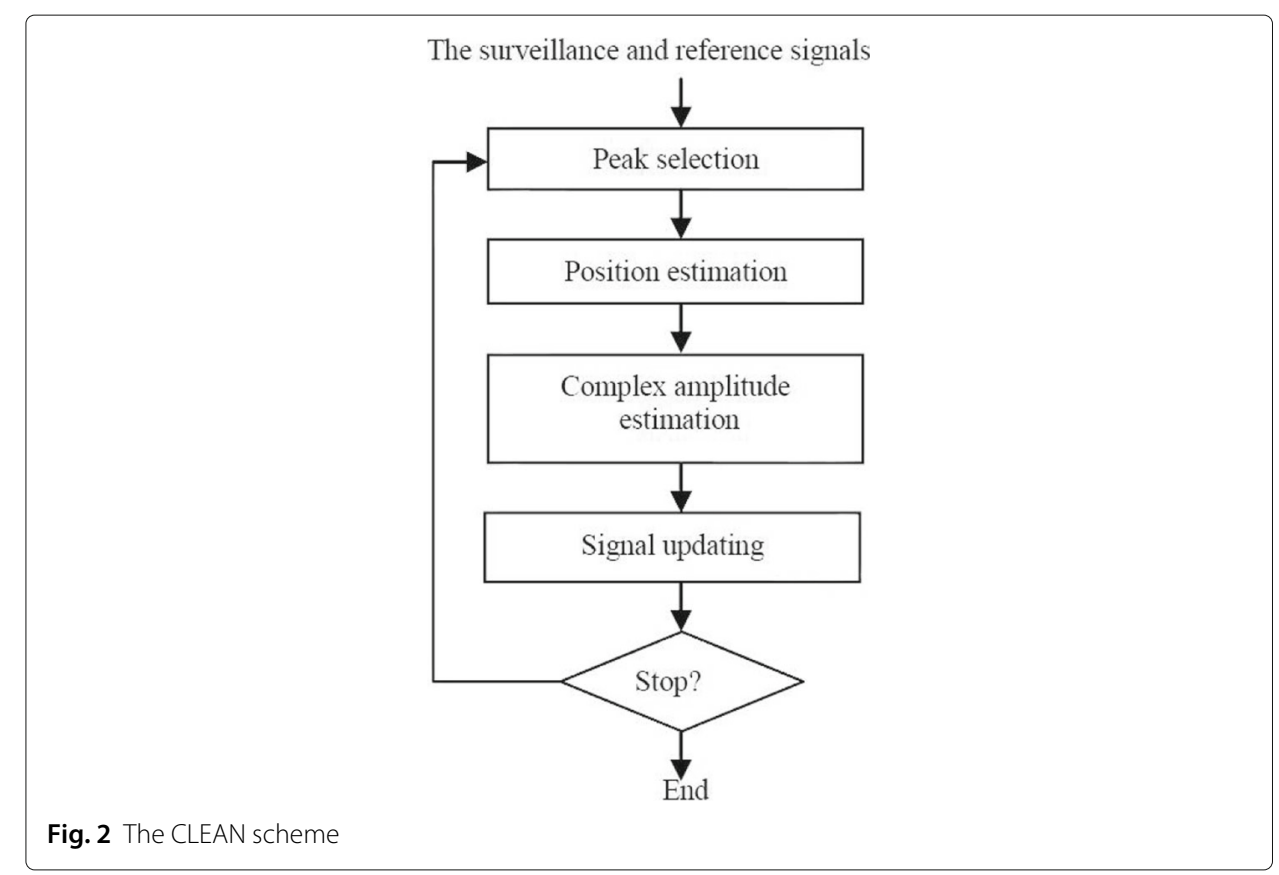

The number of coefficients is set by length $L$, that is to say, the clutter components with $i=0,1, \cdots, L-1$ has been modeled. And we can rewrite this in vector-matrix form as

$$
\boldsymbol{s}_{\text {surv }}=\boldsymbol{S}_{r} \boldsymbol{w}
$$

where $\boldsymbol{s}_{\text {surv }} \in \mathbb{C}^{N}, \boldsymbol{S}_{r} \in \mathbb{C}^{N \times L}, \boldsymbol{w} \in \mathbb{C}^{L}$ denote the observed data vector, dictionary matrix, reflective coefficient vector, respectively. They have the form

$$
\boldsymbol{s}_{\text {surv }}=\left[s_{\text {surv }}(0) s_{\text {surv }}(1) \cdots s_{\text {surv }}(N)\right]^{\mathrm{T}}
$$

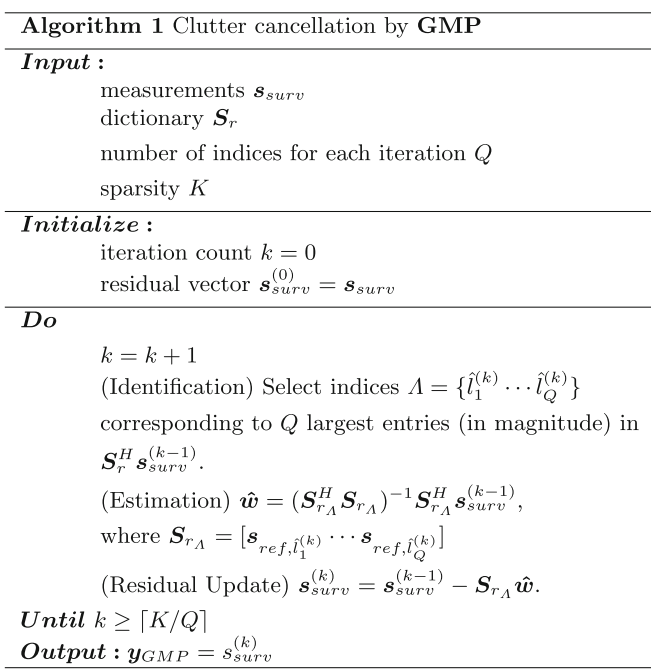

Fig. 3 Pseudocode for GMP 


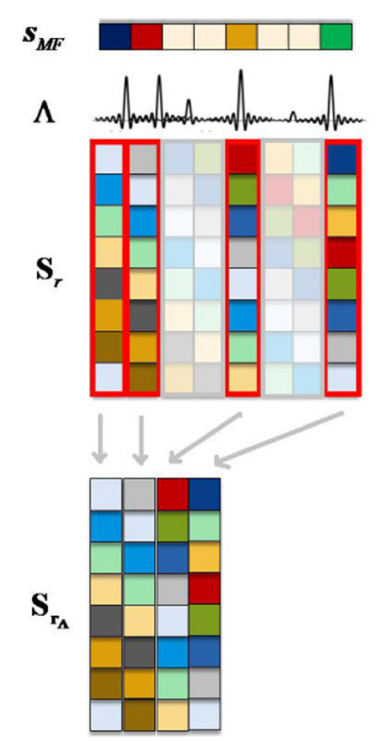

Fig. 4 Illustration for identification of batch-based CLEAN $(Q=4)$

$$
\begin{aligned}
& \boldsymbol{S}_{r}=\left[\begin{array}{llll}
s_{r e f}(0) & 0 & \cdots & 0 \\
s_{r e f}(1) & s_{r e f}(0) & \vdots \\
\vdots & s_{r e f}(1) & \ddots & 0 \\
s_{r e f}(N-L) & \vdots & & s_{r e f}(0) \\
0 & s_{r e f}(N-L) & \ddots & s_{r e f}(1) \\
\vdots & 0 & \vdots \\
0 & \cdots & 0 & s_{r e f}(N-L)
\end{array}\right] \\
& \boldsymbol{w}=\left[\begin{array}{lll}
w(0) w(1) & \cdots & w(L-1)
\end{array}\right]^{\mathrm{T}}
\end{aligned}
$$

where $N=\left\lfloor T_{\text {int }} / T_{s}\right\rfloor$ and T denotes transpose. The matrix $S_{r}$ can be written as

$$
\boldsymbol{S}_{r}=\left[\begin{array}{llll}
\boldsymbol{s}_{r e f, 0} & \boldsymbol{s}_{r e f, 1} & \cdots & \boldsymbol{s}_{r e f,(L-1)}
\end{array}\right]
$$

where $\boldsymbol{s}_{r e f, l}$ is an $N \times 1$ vector, with $l=0,1, \cdots, L-1$. In other words, the atom of dictionary matrix is time-delayed replicas of the reference waveform. The $L$-dimensional

Table 1 Computational complexity in term of complex multiplications

Processing step

$\boldsymbol{S}_{\text {ref }}=\mathrm{FFT}\left(\boldsymbol{s}_{\text {ref }, 0}\right)$

$\boldsymbol{S}_{\text {surv }}=\mathrm{FFT}\left(\boldsymbol{s}_{\text {surv }}\right)$

$\boldsymbol{s}_{\mathrm{MF}}=\operatorname{IFFT}\left(\boldsymbol{S}_{\text {surv }} \boldsymbol{S}_{\text {ref }}^{*}\right)$

$\left(\boldsymbol{S}_{r_{\Lambda}}^{H} \boldsymbol{S}_{r_{\Lambda}}\right)$

$\left(\boldsymbol{S}_{r_{\Lambda}}^{H} \boldsymbol{S}_{r_{\Lambda}}\right)^{-1}$

$\left(\boldsymbol{S}_{r_{\Lambda}}^{H} \boldsymbol{S}_{r_{\Lambda}}\right)^{-1} \boldsymbol{S}_{r_{\Lambda}}^{H} \boldsymbol{s}_{\text {surv }}$

$\boldsymbol{S}_{r_{\Lambda}}\left(\boldsymbol{S}_{r_{\Lambda}}^{H} \boldsymbol{S}_{r_{\Lambda}}\right)^{-1} \boldsymbol{S}_{r_{\Lambda}}^{H} \boldsymbol{s}_{\text {surv }}$

Total complexity
Complex multiplications

$T_{0}=N \log _{2} N / 2$

$T_{1}=N \log _{2} N / 2$

$T_{2}=N+N \log _{2} N / 2$

$T_{3}=Q^{2} N$

$T_{4}=Q^{3}$

$T_{5}=Q^{2}$

$T_{6}=Q N$

$T=T_{0}+k \sum_{i=1}^{6} T_{i}$

$k$ is the number of iterations 

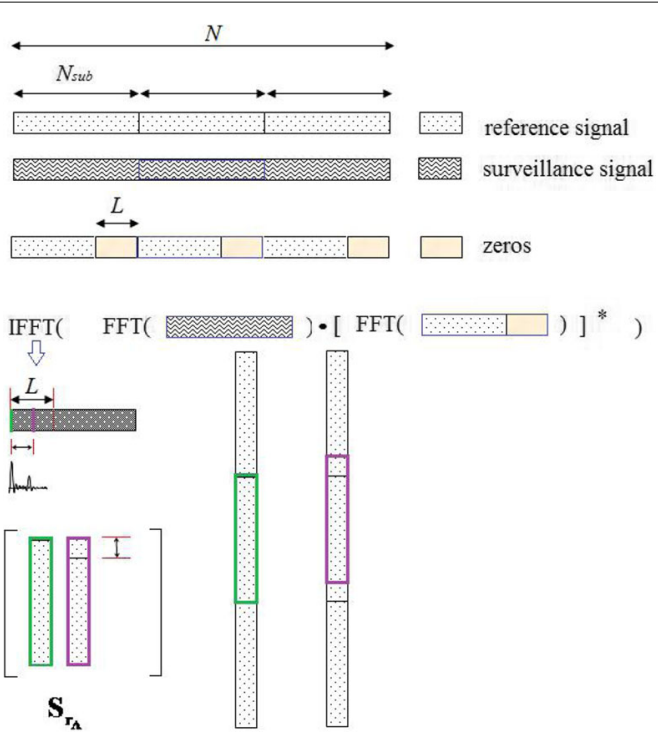

Fig. 5 Illustration for segmentation of batch-based CLEAN

coefficient vector $\boldsymbol{w}$ can be evaluated by resorting to the least square (LS) approach that minimizes the power of the output, i.e.,

$$
\hat{\boldsymbol{w}}=\left(\boldsymbol{S}_{r}^{H} \boldsymbol{S}_{r}\right)^{-1} \boldsymbol{S}_{r}^{H} \boldsymbol{s}_{\text {surv }}
$$

where $H$ denotes conjugate transpose. The $N$-dimensional output vector $\boldsymbol{y}_{L S}$ of clutter cancellation can be expressed as

$$
\boldsymbol{y}_{L S}=\boldsymbol{s}_{\text {surv }}-\boldsymbol{S}_{r} \hat{\boldsymbol{w}}
$$

\subsection{Generalized matching pursuit (GMP)}

It should be noted that the vector $\boldsymbol{w}$ is amplitude scaling from the scatterers in finite range cells. In general, there are a few dominating scatterers, so $w$ can be regarded as a sparse vector. However, when the measured signal $\boldsymbol{s}_{\text {surv }}$ contains not just clutter, but also a certain amount of noise and echo from moving target, some non-zero components $\boldsymbol{w}$ can occur to fit the non-ideal signal, which is called overfitting. Therefore, to deal with the overfitting problem, the sparse estimation of $\boldsymbol{w}$ is desirable and can be found by greedy method.

The MP algorithm is a greedy algorithm which chooses at each iteration an atom from the dictionary that is best adapted to approximate the residual signal. The absolute value of the inner product is used to measure the correlation between atoms and the residual signal. Thus, the basic CLEAN similar to MP can be regarded as projecting the

\section{Frame head (945 symbols)}

System Information (36 symbols)
Data (3744 symbols)

Fig. 6 Signal frame structure of the DTTB signal 
Table 2 Parameters of the radar system

\begin{tabular}{lll}
\hline Symbol & Quantity & Values \\
\hline$B$ & Bandwidth & $7.56 \mathrm{MHz}$ \\
$f_{s}$ & Sample frequency & $10 \mathrm{MHz}$ \\
$N$ & Sample number & 262144 \\
$\sigma_{\text {ref }}^{2}$ & Noise variance & 0.005 \\
$\sigma_{\text {surv }}^{2}$ & Noise variance & 0.005 \\
\hline
\end{tabular}

surveillance signal $\boldsymbol{s}_{\text {surv }}$ to the dictionary $\boldsymbol{S}_{r}$. The most relevant index can be obtained by

$$
\hat{l}=\underset{l}{\arg \max }\left\{\left|\left\langle\boldsymbol{s}_{\text {ref,l, }}, \boldsymbol{s}_{\text {surv }}\right\rangle\right|\right\}
$$

The surveillance signal obtained at the $k$ th iteration can be expressed as

$$
\begin{aligned}
\boldsymbol{s}_{\text {surv }}^{(k)} & =\boldsymbol{s}_{\text {surv }}^{(k-1)}-\frac{\boldsymbol{S}_{r_{\Lambda}}^{H} \boldsymbol{s}_{\text {surv }}^{(k-1)}}{\boldsymbol{S}_{r_{\Lambda}}^{H} \boldsymbol{S}_{r_{\Lambda}}} \boldsymbol{S}_{r_{\Lambda}} \\
& =\boldsymbol{s}_{\text {surv }}^{(k-1)}-\boldsymbol{S}_{r_{\Lambda}}\left(\boldsymbol{S}_{r_{\Lambda}}^{H} \boldsymbol{S}_{r_{\Lambda}}\right)^{-1} \boldsymbol{S}_{r_{\Lambda}}^{H} \boldsymbol{s}_{\text {surv }}^{(k-1)}
\end{aligned}
$$

where $\Lambda=\left\{\hat{l}^{(k)}\right\}, \boldsymbol{S}_{r_{\Lambda}}=\left[\boldsymbol{s}_{\text {ref, } \hat{l}^{(k)}}\right]$. After one or more subsequent iterations, the clutter can be suppressed and $\boldsymbol{y}_{M P}=\boldsymbol{s}_{\text {surv }}^{(k)}$ is produced.

Here, we make a direct extension of the MP by choosing indices corresponding to $Q(\geq 1)$ largest correlation in identification step of each iteration. Obviously, it embraces the MP as a special case $(Q=1)$. Therefore, our method is referred to as generalized MP (GMP), which is similar to the ideas behind GOMP [25]. GMP reduces the number of iterations and has better calculation ability. The coefficients are calculated by LS in estimation step. The clutter cancellation in passive radar can be achieved by using GMP depicted in Fig. 3. In particular, we will care more about the residual $\boldsymbol{s}_{\text {surv }}^{(k)}$ than the reconstruction performance of $\boldsymbol{w}$.

Residual update will occur continuously, and then the final residual $\boldsymbol{s}_{\text {surv }}^{(k)}$ is the cancellation output $\boldsymbol{y}_{G M P}$. Obviously, the clutter cancellation using GMP is equivalent to one using the LS when $Q=L$ (see Eq. (14)), and it is equivalent to one using the MP when $Q=1$ (see Eq. (16)).

\subsection{Batch-based CLEAN}

The key point of the proposed GMP in computation and storage exists in two steps: one is the computation of the inner product $\boldsymbol{S}_{r}^{H} \boldsymbol{s}_{\text {surv }}^{(k-1)}$, and the other is the preparation of the complete dictionary $\boldsymbol{S}_{r}$. In order to handle high-dimension signal ( $N$ is too large), we present a batch-based CLEAN technique using fast Fourier transform (FFT).

Let $\boldsymbol{S}_{\text {surv }}=\mathrm{FFT}\left(\boldsymbol{s}_{\text {surv }}\right)$ be a vector containing the Fourier transform of the surveillance signal, and $\boldsymbol{S}_{\text {ref }}=\mathrm{FFT}\left(\boldsymbol{s}_{r e f, 0}\right)$ is a vector containing the Fourier transform of the reference signal. Based on the principle of matched filtering (MF), the inner product can be obtained by $\boldsymbol{s}_{M F}=\operatorname{IFFT}\left(\boldsymbol{S}_{\text {surv }} \boldsymbol{S}_{\text {ref }}^{*}\right)$. The locations of the maximum values of $\boldsymbol{s}_{M F}$ correspond to the delay information of the strongest components of clutter, so the support set

Table 3 Parameters of the clutter

\begin{tabular}{lllllll}
\hline Clutter & Direct & Path1 & Path2 & Path3 & Path4 & Path5 \\
\hline Time delay $(i)$ & 0 & 20 & 40 & 60 & 80 & 100 \\
Amplitude $\left(W_{i}\right)$ & 1 & 0.5 & 0.4 & 0.3 & 0.1 & 0.1 \\
\hline
\end{tabular}


Table 4 Comparison of clutter cancellation methods for simulated passive radar data I

\begin{tabular}{llll}
\hline Methods & MP & GMP $(\boldsymbol{Q}=\mathbf{2})$ & GMP $(\mathbf{Q}=\mathbf{3})$ \\
\hline Cancellation gain $(\mathrm{dB})$ & 33.792 & 34.0561 & 34.2929 \\
Run time $(\mathrm{s})$ & 0.7620 & 0.4300 & 0.3070 \\
\hline
\end{tabular}

$\Lambda$ and the corresponding partial dictionary $S_{r_{\Lambda}}$ can be determined, which is shown in Fig. 4. Due to the fact that the batch-based CLEAN method directly constructs a matrix $\boldsymbol{S}_{r_{\Lambda}}^{N \times Q}$ of order $Q$ without building a dictionary $\boldsymbol{S}_{r}^{N \times L}$ of order $L$ (where $Q \ll L$ ), this method greatly reduces the requirements on storage space. We analyze the computational complexity of the proposed batch-based CLEAN algorithm. Table 1 summarizes the computational complexity in term of complex multiplications.

The CLEAN-type technique usually assumes that time-invariant clutter environment over the CPI, which may not hold in practice. In response to the time-variant characteristic of clutter, we present a batch-based CLEAN with segmentation. In our method, the recorded reference signal and surveillance signal are first divided into data segments with length of $N_{s u b}$. In each signal segment, the amplitude and the time delay of clutter can be regarded as nearly constant. The next step is zero setting to the last $L$ elements at the end of reference segment, and then FFT-based matched filtering is implemented to process the surveillance segment. The previous $L$ elements in the following output vector can then be used in the identification of batch-based CLEAN. The illustration for proposed processing is shown in Fig. 5. One thing to note is that all segments can be processed in parallel.

\section{Experimental results and discussion}

In this section, we present experimental results with simulated data and real data. The performance of the developed batch-based CLEAN technique is here analyzed.

\subsection{Simulated data}

We have conducted numerical experiments to investigate the performance of the proposed clutter cancellation method. The reference channel signal of digital television (DTV)-based passive radar is simulated. Chinese DTV standard (digital television terrestrial broadcasting (DTTB)) was published in 2006 [26]. The signal frame structure is given in Fig. 6, which explains that the signal frame consists of frame head (945 symbols) and frame body (3780 symbols). For surveillance channel signal simulation, we assume that there is no moving target. The radar and clutter parameters used in the simulation are listed in Tables 2 and 3. Thermal noise was modeled as complex Gaussian white noise.

In order to quantitatively evaluate the performances of clutter cancellation methods, we use cancellation gain as a metric. The definition of gain $G_{c}$ is

$$
G_{c}=10 \log _{10} \frac{\left\|\boldsymbol{s}_{\text {surv }}^{(0)}\right\|_{2}^{2}}{\left\|\boldsymbol{s}_{\text {surv }}^{(k)}\right\|_{2}^{2}}
$$

Table 5 Comparison of clutter cancellation methods with FFT for simulated passive radar data

\begin{tabular}{llll}
\hline Methods & Basic CLEAN & Batch-based CLEAN $(\boldsymbol{Q}=\mathbf{2})$ & Batch-based CLEAN $(\mathbf{Q}=\mathbf{3})$ \\
\hline Cancellation gain $(\mathrm{dB})$ & 33.792 & 34.0561 & 34.2929 \\
Run time $(\mathrm{s})$ & 0.2330 & 0.1600 & 0.1300 \\
\hline
\end{tabular}




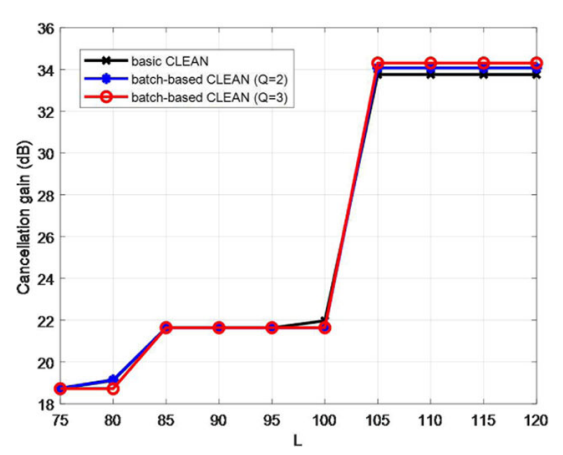

Fig. 7 The effect of parameter $L$ on cancellation gain

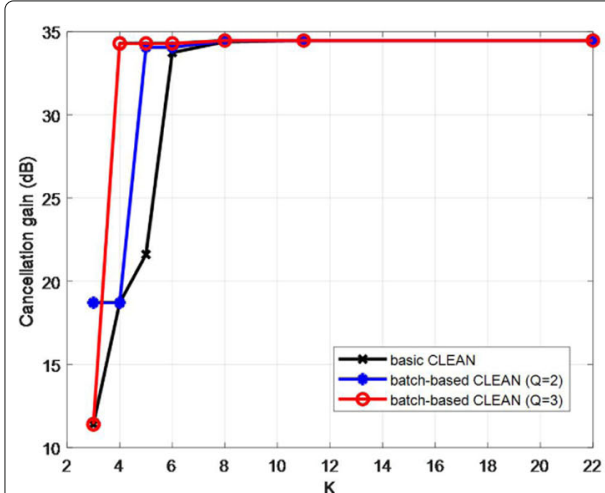

(a)

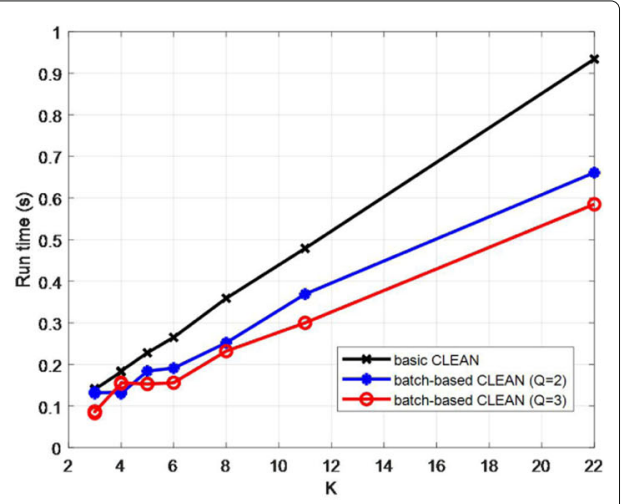

(b)

Fig. 8 The effect of the termination criterion Eq. (18). a Cancellation gain vs. parameter $K$. b Run time vs. parameter $K$

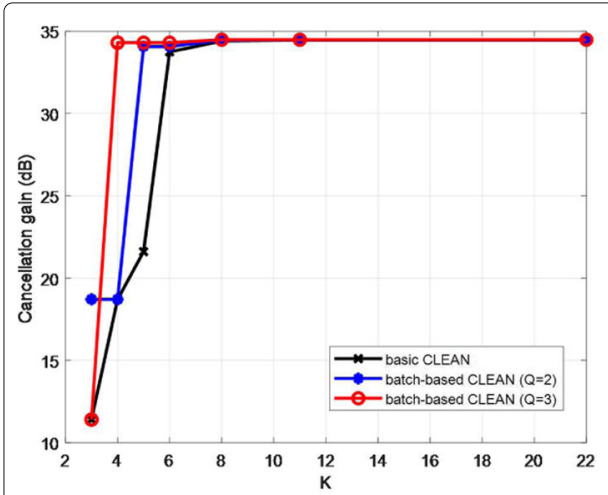

(a)

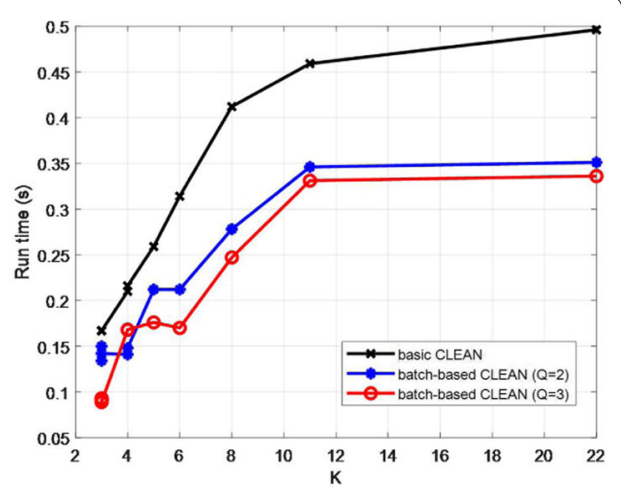

(b)

Fig. 9 The effect of the termination criterion Eq. (19). a Cancellation gain vs. parameter K. b Run time vs. parameter $K$ 


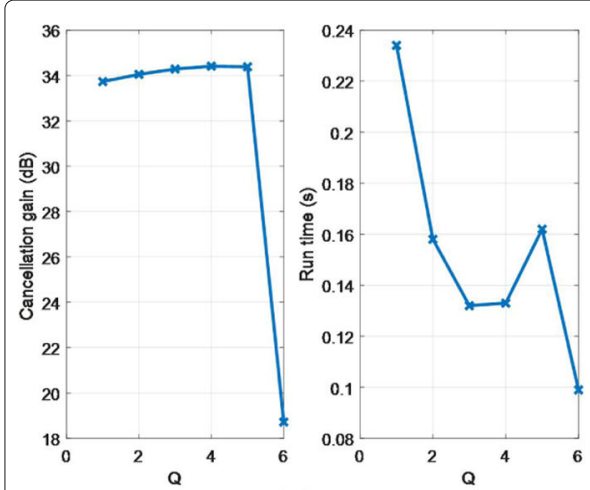

(a)

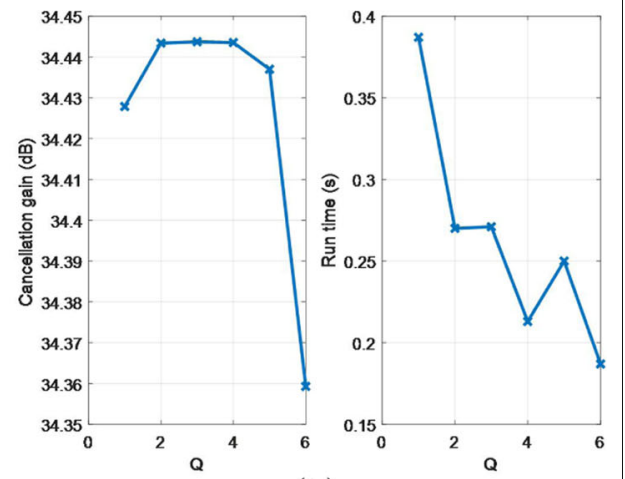

(b)

Fig. 10 The effect of parameter $Q$ on cancellation gain and run time. $\mathbf{a} K=6 . \mathbf{b} K=12$

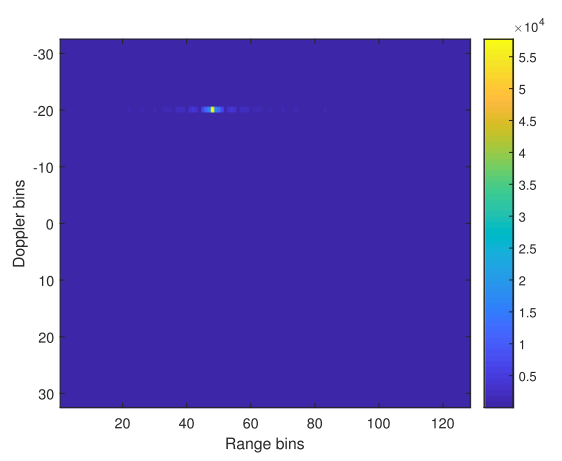

Fig. 11 The RD map of a moving target

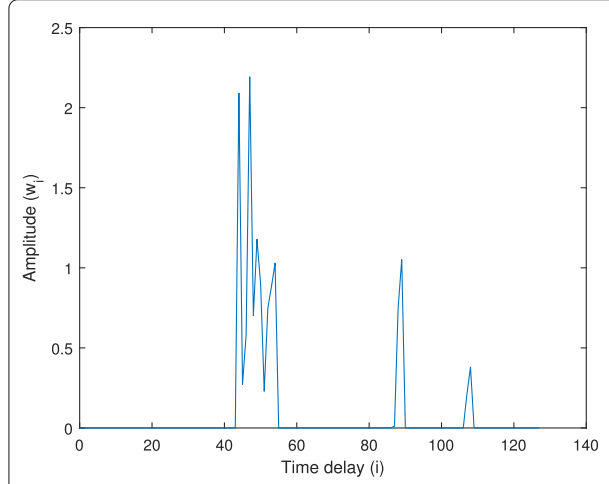

(a)

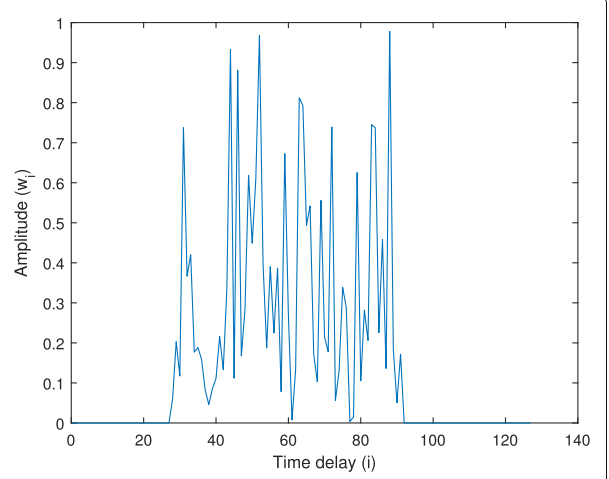

(b)

Fig. 12 The diagram of distributed clutter: $\mathbf{a}$ case A and $\mathbf{b}$ case B 


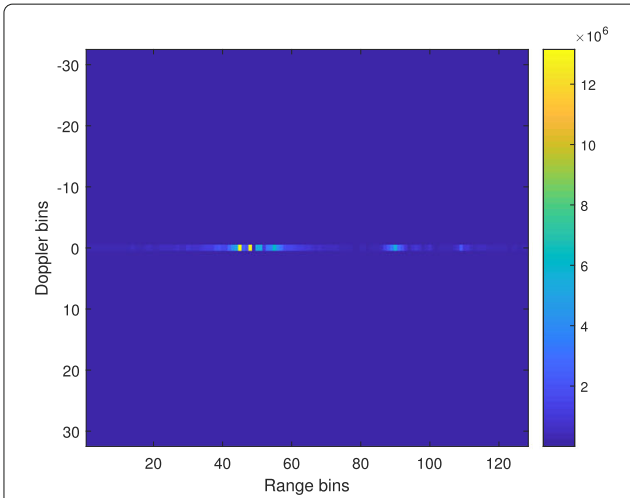

(a)

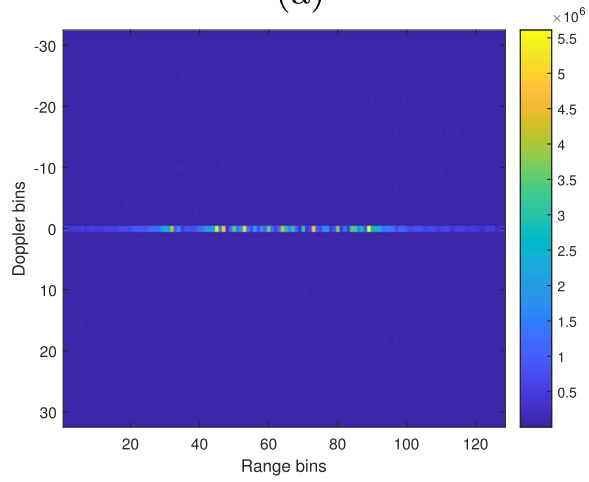

(c)

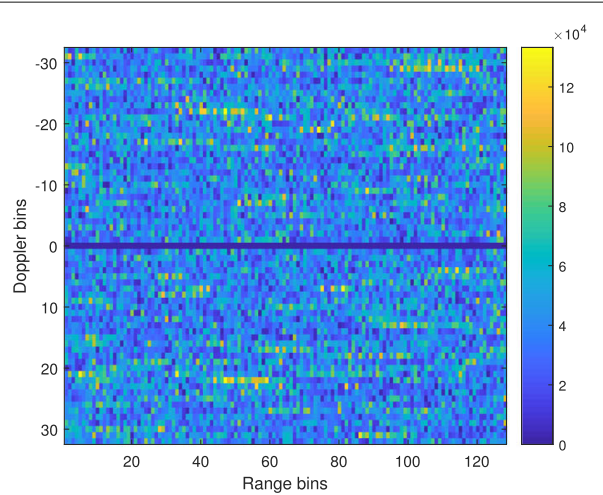

(b)

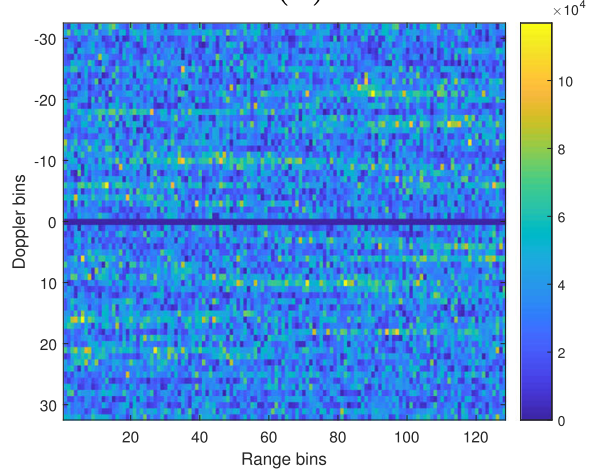

(d)

Fig. 13 The RD maps: $\mathbf{a}$ before and $\mathbf{b}$ after masking zero-Doppler for case A; $\mathbf{c}$ before and $\mathbf{d}$ after masking zero-Doppler for case B

In general, the larger cancellation gain implies better clutter cancellation. The performances of clutter cancellation methods $(L=128, K=6)$ are shown in Table 4. The run times are shown in the final row. Owing to the selection of multiple time-delayed replicas, the GMP is finished with smaller number of iterations when compared to the MP. The results show that the method by using proposed GMP is well done. It has good cancellation performance with fast processing speed. To enhance the feasibility of this clutter cancellation method, FFT is adopted to accomplish the speediness calculation, which is listed in Table 5.

It should be noted that the main parameters to be selected are $L, K$, and $Q$, which affects the clutter cancellation. We must note that the optimal values are environment specific. Parameters will be set empirically for the data at hand. It needs to follow the below principles.

(i) The best $L$ is exactly large enough to contain principal components of clutter. Figure 7 shows that smaller $L$ results in decreased cancellation gain, while bigger $L$ cannot achieve

Table 6 Comparison of cancellation gains

\begin{tabular}{lllll}
\hline Methods & SMI (LS) & Basic CLEAN & Batch-based CLEAN $(\boldsymbol{Q}=\mathbf{2})$ & Batch-based CLEAN $(\mathbf{Q}=\mathbf{3})$ \\
\hline Case A & $46.4314 \mathrm{~dB}$ & $29.5240 \mathrm{~dB}$ & $29.8365 \mathrm{~dB}$ & $30.6290 \mathrm{~dB}$ \\
Case B & $44.6188 \mathrm{~dB}$ & $19.1719 \mathrm{~dB}$ & $19.4983 \mathrm{~dB}$ & $20.0736 \mathrm{~dB}$ \\
\hline
\end{tabular}


Table 7 Comparison of run times

\begin{tabular}{lllll}
\hline Methods & SMI (LS) & Basic CLEAN & Batch-based CLEAN $(\boldsymbol{Q}=\mathbf{2})$ & Batch-based CLEAN $(\mathbf{Q}=\mathbf{3})$ \\
\hline Case A & $6.3280 \mathrm{~s}$ & $1.9000 \mathrm{~s}$ & $1.7450 \mathrm{~s}$ & $1.2050 \mathrm{~s}$ \\
Case B & $6.7970 \mathrm{~s}$ & $4.5110 \mathrm{~s}$ & $3.3500 \mathrm{~s}$ & $2.1840 \mathrm{~s}$ \\
\hline
\end{tabular}

further improvements of cancellation gain. In the actual application, the value of $L$ is usually set according to the scene of indoor or outdoor surveillance.

(ii) $K$ indicates sparsity of clutter, which is the number of principal components and is typically unknown. The termination criterion of the proposed batch-based CLEAN method is

$$
k \geq\lceil K / Q\rceil
$$

Obviously, the bigger sparsity $K$ means more iterations. As the sparsity increases, the cancellation gain increases firstly and tends to be stable finally, but the run time increases continuously. The results are shown in Fig. 8. Therefore, using the variation of cancellation gain, the termination criterion is modified as

$$
k \geq\lceil K / Q\rceil \text { or }\left|G_{c}^{(k)}-G_{c}^{(k-1)}\right|<T h
$$

where $G_{c}^{(k)}$ and $G_{c}^{(k-1)}$ are cancellation gains in adjacent iterations and $T h$ is a threshold. Obviously, a small variation of cancellation gain means that clutter cancellation is almost complete. Figure 9 shows the results using new termination criterion $(T h=0.1)$, which can reduce unnecessary time consumption.

(iii) $Q$ is not the larger the better. It is true that the number of iterations usually decreases for larger $Q$ values, but the amount of calculation and storage in each iteration are larger simultaneously. Moreover, bigger $Q$ may cause the selection of "incorrect" indices. Figure 10a demonstrates the degradation in cancellation gain when $Q=6$. However, this is not a serious problem, which can be solved through the addition of iterations(see Fig. 10b). Thus, there is a tradeoff decided in accordance with actual condition.

To further evaluate the performance of the proposed cancellation technique, the following simulated scenario will consist of one moving target echo and stationary distributed clutter. The moving target has the following parameters. The amplitude ratio $A_{m t} / A$ is 0.01 , the range cell index $\left(\tau_{m t}\right)$ is 47 , and the Doppler cell index $\left(f_{m t}\right)$ is -20 . The other parameters are set to the same as that in previous simulated experiment. In an ideal situation (no clutter), the range-Doppler (RD) map obtained by the classical CAF is shown in Fig. 11. We select two specific clutter cases as reported in Fig. 12a and b. Case A and case B represent the moderate clutter and the severe clutter, respectively. And then, the RD maps obtained by the classical CAF are shown in Fig. 13. In the left figures, only zero-Doppler components are visible. It can be found from the right figures that the non-zero-Doppler component related to the moving target is still invisible after masking zero-Doppler.

Table 8 Comparison of signal-to-noise ratios

\begin{tabular}{llllll}
\hline Methods & No clutter & SMI (LS) & Basic CLEAN & $\begin{array}{l}\text { Batch-based } \\
\text { CLEAN }(\boldsymbol{Q}=\mathbf{3})\end{array}$ & $\begin{array}{l}\text { Batch-based } \\
\text { CLEAN (Q = 2) }\end{array}$ \\
\hline Case A & $48.7937 \mathrm{~dB}$ & $37.7820 \mathrm{~dB}$ & $30.8026 \mathrm{~dB}$ & $30.9725 \mathrm{~dB}$ & $31.7082 \mathrm{~dB}$ \\
Case B & $48.7937 \mathrm{~dB}$ & $34.3216 \mathrm{~dB}$ & $22.7736 \mathrm{~dB}$ & $22.9700 \mathrm{~dB}$ & $23.5549 \mathrm{~dB}$ \\
\hline
\end{tabular}




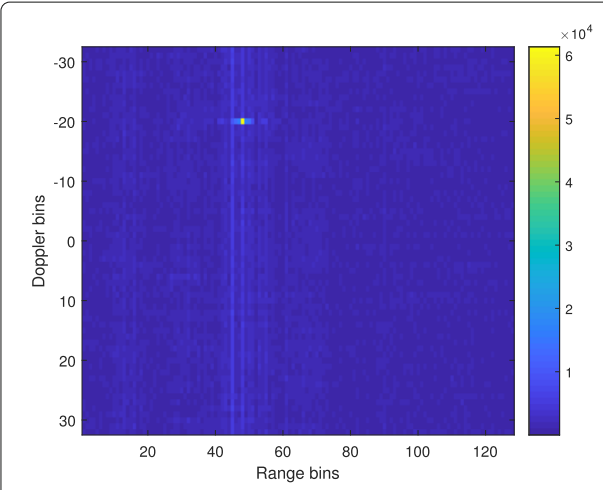

(a)

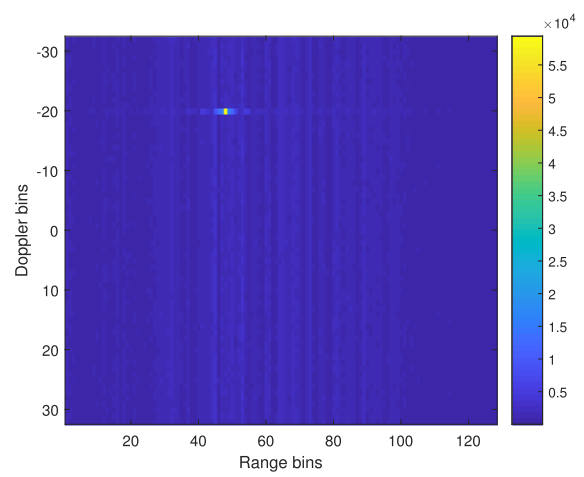

(c)

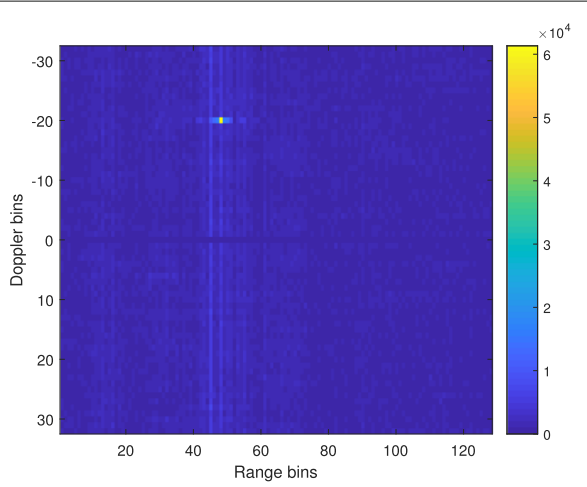

(b)

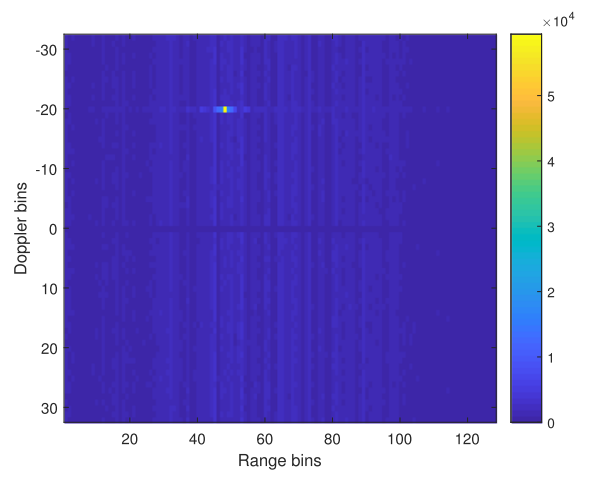

(d)

Fig. 14 The RD maps obtained by using SMl $\mathbf{a}$ before and $\mathbf{b}$ after masking zero-Doppler for case $A ; \mathbf{c}$ before and $\mathbf{d}$ after masking zero-Doppler for case B

And then, we compare the proposed batch-based CLEAN method with basic CLEAN and SMI. The performance analysis of clutter cancellation methods is shown in Tables 6, 7, and 8, which correspond to cancellation gains, run times, and signal-to-noise ratios (SNRs), respectively. As is expected, the SMI method has a good cancellation capability, while it uses a longer running time than other CLEAN methods. For the two clutter cases, the CLEAN technique is still useful, but the performance has an obvious degradation. Compared with the basic CLEAN method, the proposed batch-based CLEAN has a better capability.

The RD maps obtained by using SMI are presented in Fig. 14. It is clear that SMI exhibited high level of cancellation. Figure 15 depicts the results obtained by using batch-based CLEAN. These figures show that the CLEAN method has acceptable performances in terms of clutter cancellation. In other words, the moving target becomes visible, though the residual clutter is also visible. It is important to point out that the SMI method has to construct the complete dictionary (see Eq. (10)), and there will be a great space use. Because of high memory cost of the SMI method, it cannot be implemented in subsequent experiment. Owing to parameter $Q$, the proposed method leads to greater flexibility.

\subsection{Real data}

The effectiveness of the proposed CLEAN technique with segmentation is demonstrated using a real data set derived from an experimental DTV-based PR system. The scene of 


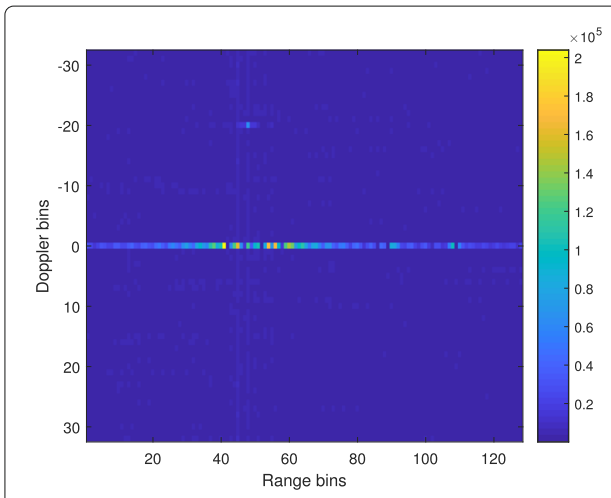

(a)

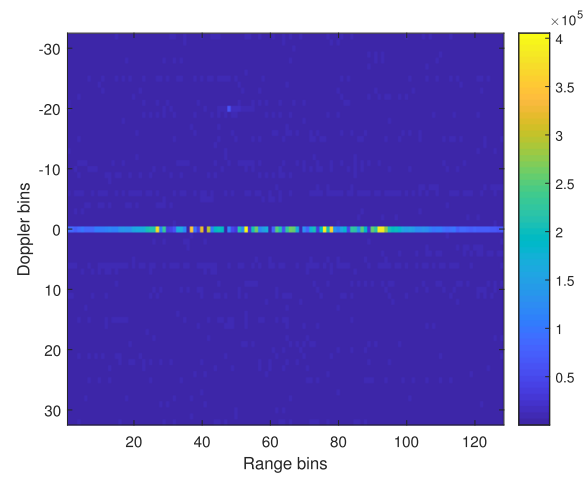

(c)

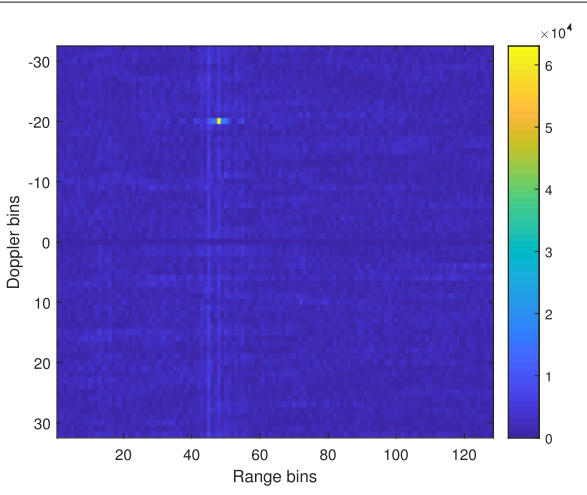

(b)

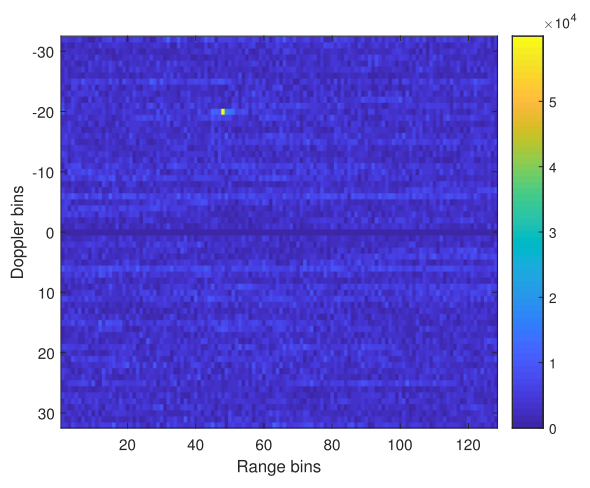

(d)

Fig. 15 The RD maps obtained by using the proposed method $(Q=3) \mathbf{a}$ before and $\mathbf{b}$ after masking zero-Doppler for case A; $\mathbf{c}$ before and $\mathbf{d}$ after masking zero-Doppler for case B

data collection is shown in Fig. 16. The illuminator of opportunity considered in this paper is a terrestrial TV transmitter located in Beijing, China. The receiver is located on top of the library building in the LiangXiang campus of Beijing Institute of Technology (BIT). The experiment was conducted near Nanyuan Airport. The carrier frequency is $674 \mathrm{MHz}$, the bandwidth is $7.56 \mathrm{MHz}$, and the sampling rate is $10 \mathrm{MHz}$.

The data set used in this paper is a 200-ms capture including an airborne target and several stationary targets. The CAF result without clutter cancellation is shown in Fig. 17, and the target is completely masked by sidelobes of clutter. The data has been processed

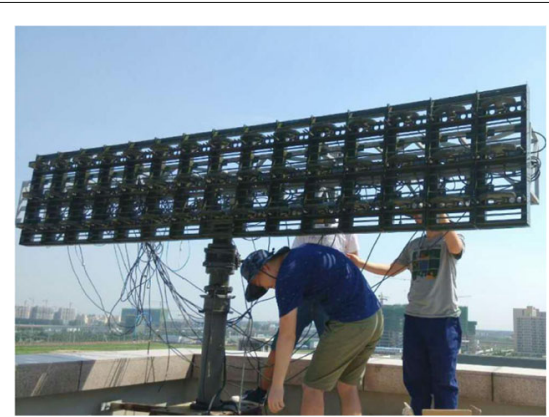

Fig. 16 DTV-based PR system 


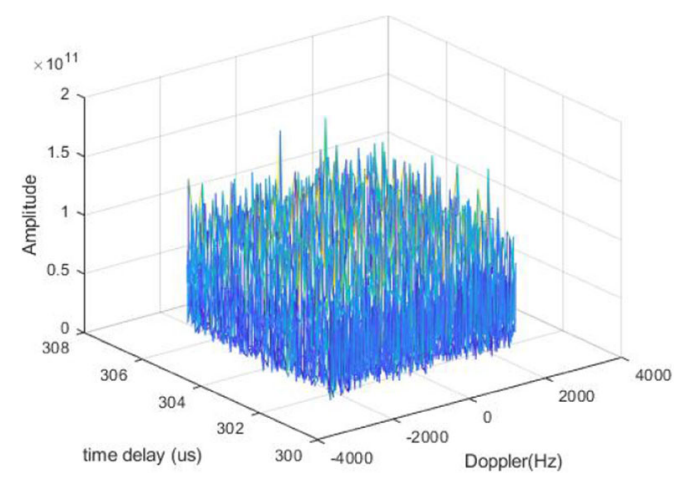

Fig. 17 The CAF result without clutter cancellation

offline by the proposed batch-based CLEAN with segmentation. Each segment of data takes the same time and can all be processed in parallel. The sample number of each segment $N_{\text {sub }}$ will ensure that at least the output of matched filtering (MF) maintains an enough high correlation peak to ensure we get correct identification information. Here, we set main parameters as follows: $N_{\text {sub }}=65536, L=4096$.

Figure 18 illustrates the effect of sparsity setting to cancellation gain and run time obtained by using basic CLEAN. According to the obtained result, we can conclude that there is dense clutter in the experimental scene (urban scene). We should set the parameter $K$ in a reasonable range $L / 2 \leq K \leq L$. The performances of the batch-based CLEAN are demonstrated in Fig. 19. As the value of $Q$ increases, the cancellation gain has increasing trend, but the run time decreases firstly and increases finally, so $Q$ cannot be too large. The results show that the proposed clutter cancellation method $(Q>1)$ has not only accelerated the computational speed, but also improved cancellation gain.

After clutter cancellation, the coherent integration results are shown in Figs. 20 and 21, where the peak of the moving target is obvious. It can be seen that the moving target is submerged in the clutter signal before clutter cancellation, and the target can be detected after CLEAN processing. The SNRs of CAF results are listed in Table 9, which shows the proposed batch-based CLEAN technique gives better detection performance for passive radar.
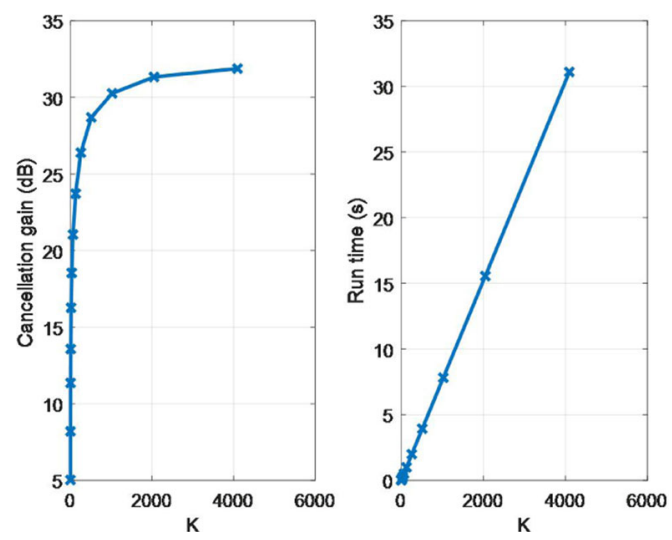

Fig. 18 The effect of parameter $K$ to cancellation gain and run time obtained by using basic CLEAN 

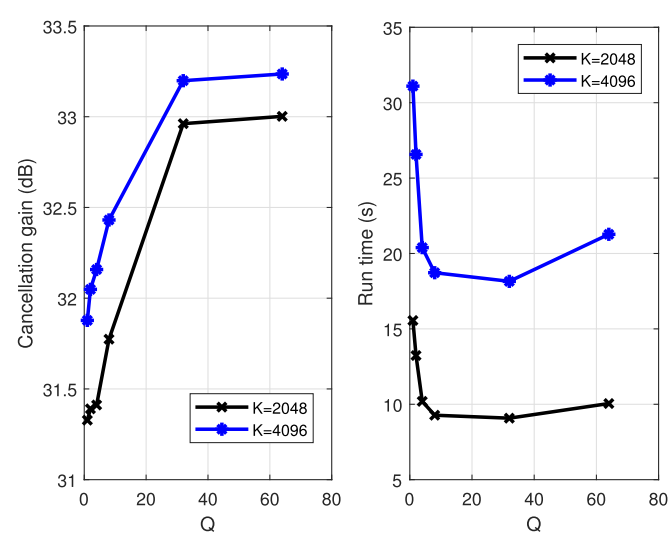

Fig. 19 The performances of batch-based CLEAN

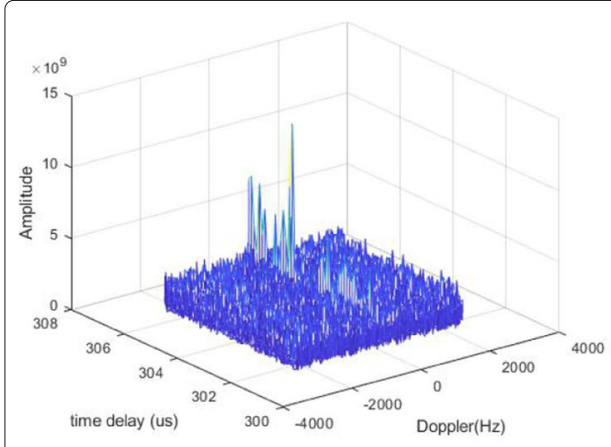

(a)

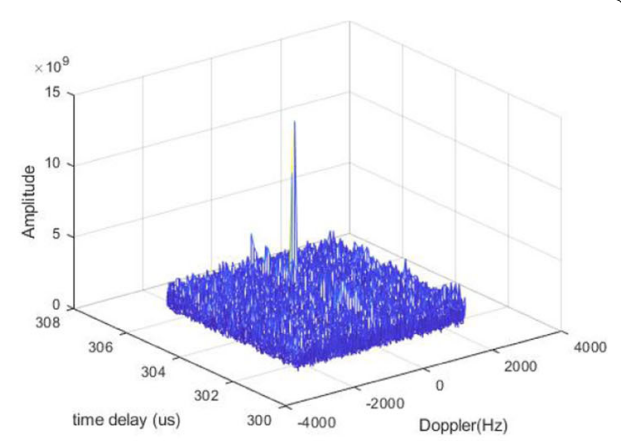

(b)

Fig. 20 The CAF results on real data after a basic CLEAN $(K=2048)$ and $\mathbf{b}$ batch-based CLEAN $(K=2048$, $Q=64)$

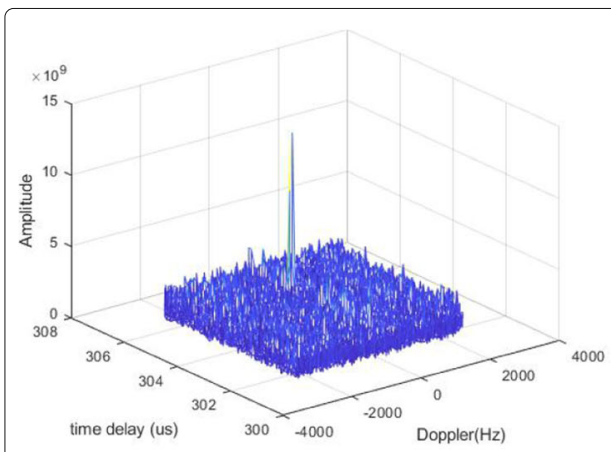

(a)

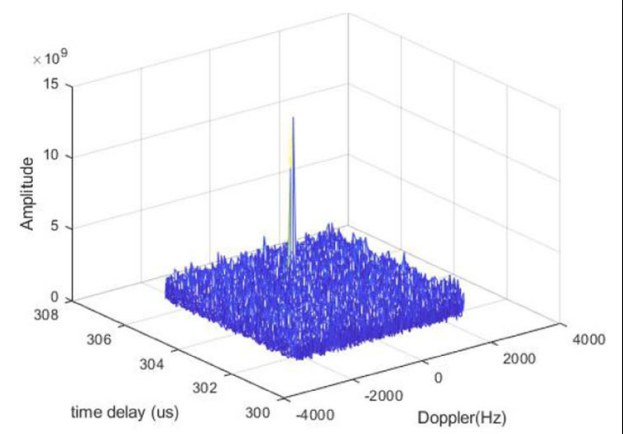

(b)

Fig. 21 The CAF results on real data after a basic CLEAN $(K=4096)$ and $\mathbf{b}$ batch-based CLEAN $(K=4096$, $Q=64$ ) 
Table 9 Signal-to-noise ratios of CAF

\begin{tabular}{lll}
\hline Methods & Basic CLEAN & Batch-based CLEAN $(\boldsymbol{Q}=\mathbf{6 4})$ \\
\hline$K=2048$ & $20.4372 \mathrm{~dB}$ & $22.7300 \mathrm{~dB}$ \\
$K=4096$ & $21.2766 \mathrm{~dB}$ & $23.5982 \mathrm{~dB}$ \\
\hline
\end{tabular}

\title{
5 Conclusion
}

In passive radar, clutter cancellation is a key problem for weak signal detection, and the CLEAN algorithm is used for clutter cancellation. To speed up the computational processing and improve the cancellatio4 gain, a batch-based CLEAN using GMP and FFT is proposed. Furthermore, the effects of various parameters on performance are analyzed. Experiment results show that through the proposed scheme, the clutter is effectively suppressed and the weak target emerges. The batch-based CLEAN technique can serve as a good candidate for clutter cancellation in passive radar.

\begin{abstract}
Abbreviations
PR: Passive radar; LMS: Least mean square; RLS: Recursive least square; MP: Matching pursuit; GMP: Generalized matching pursuit; FFT: Fast fourier transform; LS: Least square; CAF: Cross ambiguity function CAF; CPI: Coherent processing interval; GOMP: Generalized orthogonal matching pursuit; DTV: Digital television; DTTB: Digital television terrestrial broadcasting; SNRs: Signal-to-noise ratios
\end{abstract}

\section{Acknowledgements}

The authors are grateful to the National Science Foundation of China for its support of this research.

\section{Authors' contributions}

X. Bai and J. Han designed and performed the experiments. And then XB analyzed the results and wrote the manuscript. All authors discussed the results and revised the manuscript. The author(s) read and approved the final manuscript.

\section{Funding}

This work was supported in part by the National Natural Science Foundation of China (Grant 61771046, 61731023, 61421001).

\section{Availability of data and materials}

The data that support the findings of this study are available from the corresponding author upon reasonable request.

\section{Competing interests}

The authors declare that they have no competing interests.

Received: 29 January 2021 Accepted: 15 July 2021

Published online: 10 August 2021

\section{References}

1. G. Reitsma, FM radio based bistatic radar. IEE Proc.- Radar, Sonar Navig. 152(3), 107-115 (2005)

2. H. D. Griffiths, C. J. Baker, Passive coherent location radar systems. Part 1: performance prediction. IEE Proc.- Radar, Sonar Navig. 152(3), 153-159 (2005)

3. C. J. Baker, H. D. Griffiths, I. Papoutsis, Passive coherent location radar systems. Part 2: waveform properties. IEE Proc.Radar, Sonar Navig. 152(3), 160-168 (2005)

4. H. Kuschel, in Proc. International Conference on Radar, Approaching 80 years of passive radar (IEEE, Adelaide, 2013), pp. 213-217

5. K. E. Olsen, W. Asen, Bridging the gap between civilian and military passive radar. IEEE Aerosp. Electron. Syst. Mag. 32(2), 4-12 (2017)

6. J. L. Garry, G. E. Smith, Passive ISAR part I: framework and considerations. IET Radar, Sonar Navig. 13(2), 169-180 (2019)

7. A. Manno-Kovacs, E. Giusti, F. Berizzi, L. Kovács, Image based robust target classification for passive ISAR. IEEE Sensors J. 19(1), 268-276 (2019)

8. M. Malanowski, in Proc. International Radar Symposium, Comparison of adaptive methods for clutter removal in PCL radar (IEEE, Krakow, 2006), pp. 1-4

9. R. Cardinali, F. Colone, C. Ferretti, P. Lombardo, in Proc. IEEE Radar Conf. (RadarConf), Comparison of clutter and multipath cancellation techniques for passive radar (IEEE, Boston, 2007), pp. 469-474

10. J. E. Palmer, S. J. Searle, in Proc. IEEE Radar Conf. (RadarConf), Evaluation of adaptive filter algorithms for clutter cancellation in passive bistatic radar (IEEE, Atlanta, 2012), pp. 493-498

11. J. L. Garry, G. E. Smith, C. J. Baker, in Proc. Signal Process. Symp. (SPSympo), Direct signal suppression schemes for passive radar (IEEE, Debe, 2015), pp. 1-5

12. T. Peto, R. Seller, in Proc. International Radar Symposium (IRS), Time domain filter comparison in passive radar systems (IEEE, Prague, 2017), pp. 1-10 
13. V. J. Schwarz, Mathematical-statistical description of the iterative beam removing technique (method CLEAN). Astron. Astrophys. 65, 345-356 (1978)

14. A. Segalovitz, B. R. Friedene, A CLEAN-type deconvolution algorithm. Astron. Astrophys. 70, 335-343 (1978)

15. K. Kulpa, P. Samczynski, M. Malanowski, L. Maslikowski, V. Kubica, in Proc. IEEE Radar Conf. (RadarConf), The use of CLEAN processing for passive SAR image creation (IEEE, Ottawa, 2013), pp. 1-6

16. L. Maslikowski, P. Samczynski, M. Baczyk, P. Krysik, K. Kulpa, Passive bistatic SAR imaging—challenges and limitations. IEEE Aerosp. Electron. Syst. Mag. 29(7), 23-29 (2014)

17. D. Gromek, K. Kulpa, P. Samczynski, Experimental results of passive SAR imaging using DVB-T illuminators of opportunity. IEEE Geosci. Remote Sens. Lett. 13(8), 1124-1128 (2016)

18. K. S. Kulpa, Z. Czekała, Masking effect and its removal in PCL radar. IEE Proc.-Radar, Sonar Navig. 152(3), 174-178 (2005)

19. J. Palmer, S. Palumbo, A. Summers, D. Merrett, S. Searle, S. Howard, An overview of an illuminator of opportunity passive radar research project and its signal processing research directions. Digit. Sig. Process. 21(5), 593-599 (2011)

20. S. G. Mallat, Z. Zhang, Matching pursuits with time-frequency dictionaries. Trans. Acoust., Speech, Sig. Process. 41(12), 3397-3415 (1993)

21. S. F. Cotter, B. D. Rao, Sparse channel estimation via matching pursuit with application to equalization. IEEE Trans. Commun. 50(3), 374-377 (2002)

22. J. Bosse, O. Rabaste, Subspace rejection for matching pursuit in the presence of unresolved targets. IEEE Trans. Sig Process. 66(8), 1997-2010 (2018)

23. P. Krysik, Z. Gajo, in Proc. European Radar Conference, The impact and minimization of multipath propagation effects in the reference channel of a GSM based passive radar (IEEE, Rome, 2014), pp. 116-120

24. X. Zhang, J. Yi, X. Wan, Y. Liu, in Proc.IEEE Radar Conference, On the performance of multipath in reference signal for passive radar interference cancellation (IEEE, Seattle, 2017), pp. 1313-1316

25. J. Wang, S. Kwon, B. Shim, Generalized orthogonal matching pursuit. IEEE Trans. Sig. Process. 60(12), 6202-6216 (2012)

26. J. Feng, J. Liu, Zhou X., GB (Chinese National Standard) 20600-2006:'Framing structure, channel coding and modulation for digital television terrestrial broadcasting system[J], SAC (Standardization Administration of the People's Republic of China)' (2006)

\section{Publisher's Note}

Springer Nature remains neutral with regard to jurisdictional claims in published maps and institutional affiliations.

\section{Submit your manuscript to a SpringerOpen ${ }^{\circ}$ journal and benefit from:}

- Convenient online submission

Rigorous peer review

- Open access: articles freely available online

- High visibility within the field

- Retaining the copyright to your article

Submit your next manuscript at $\boldsymbol{\triangleright}$ springeropen.com 